\title{
Therapeutic effects of oxaliplatin-based neoadjuvant chemotherapy and chemoradiotherapy in patients with locally advanced rectal cancer: a single-center, retrospective cohort study
}

Takashi Okuyama1*, Shinichi Sameshima', Emiko Takeshita', Ryuji Yoshioka', Yukinori Yamagata', Yuko Ono², Nobumi Tagaya ${ }^{1}$, Tamaki Noie ${ }^{1}$ and Masatoshi Oya ${ }^{1}$

\begin{abstract}
Background: Neoadjuvant chemoradiotherapy (NACRT) has now become the standard treatment for locally advanced rectal cancer (LARC). NACRT has decreased local relapse (LR) rate in patients with LARC; however, distant relapse has recently attracted much attention. This study aimed to assess the feasibility and efficiency of neoadjuvant chemotherapy (NAC) for LARC.
\end{abstract}

Methods: Data on patients with cT3/4 and N+ rectal cancer who were treated in our institution from April 2010 to February 2016 were reviewed retrospectively. Twenty-seven patients who received 2-9 cycles of oxaliplatin-based NAC and 28 patients who received NACRT (45 Gy delivered in 25 fractions and 5 -fluorouracil-based oral chemotherapy) were analyzed. The primary and secondary endpoints of the present study were the 3-year relapse-free survival (RFS) and the local and distant relapse rates, respectively.

Results: Regardless of the kind of neoadjuvant therapy, no patient experienced any grade 3-4 therapy-related adverse events. The frequent toxic events were grade 1 diarrhea in patients with NACRT and neutropenia in patients with NAC. A significantly higher proportion of patients with NAC underwent laparoscopic surgery and anterior resection $(p=0.037$ and $p=0.003$, respectively). The percentages of patients with lymph node yield less than 12 in the NAC group, and those in the NACRT group were 26 and $68 \%$, respectively $(p=0.002)$. Comparing the NAC with the NACRT groups, the local relapse and distant relapse rates were 7.4 and $7.1 \%$ and 7.4 and $18 \%$, respectively. There were no significant differences in 3-year RFS and 4-year overall survival (OS) between NAC and NACRT (3-year RFS 85.2 vs. 70.4\%, $p=0.279$; 4-year OS 96.3 vs. $89.1 \%, p=0.145$, respectively). With an analysis excluding patients who received postoperative adjuvant chemotherapy, no patients who received NAC had a distant relapse, and there was a significant difference in 3-year RFS compared with the NACRT groups ( 94.4 vs. $63.2 \%, p=0.043$ ).

Conclusion: These outcomes suggest that the therapeutic effect of oxaliplatin-based NAC is at least equal to that of NACRT and that NAC is a feasible and promising option for LARC.

Keywords: Locally advanced rectal cancer, Oxaliplatin-based neoadjuvant chemotherapy, Neoadjuvant chemoradiotherapy, Relapse-free survival

\footnotetext{
*Correspondence: okuyama615@gmail.com

'Department of Surgery, Dokkyo Medical University, Koshigaya Hospital,

2-1-50, Minami Koshigaya, Koshigaya, Saitama 343-8555, Japan

Full list of author information is available at the end of the article
}

(c) The Author(s). 2018 Open Access This article is distributed under the terms of the Creative Commons Attribution 4.0 International License (http://creativecommons.org/licenses/by/4.0/), which permits unrestricted use, distribution, and reproduction in any medium, provided you give appropriate credit to the original author(s) and the source, provide a link to the Creative Commons license, and indicate if changes were made. The Creative Commons Public Domain Dedication waiver (http://creativecommons.org/publicdomain/zero/1.0/) applies to the data made available in this article, unless otherwise stated. 


\section{Background}

Therapeutic strategies for locally advanced rectal cancer (LARC) have evolved significantly during the last three decades. In Western countries, neoadjuvant chemoradiotherapy (NACRT) followed by total mesorectal excision (TME) is currently the standard treatment option for patients with LARC [1]. However, it is well known that, although NACRT reduces the local recurrence to a rate less than $10 \%$, distant relapse occurs in $25-40 \%$ of patients [2-4]. This distant relapse rate is almost equal to the local relapse rate in patients who receive no neoadjuvant therapy (NAT) [5]. In addition, several patients who received NACRT had an unfavorable response, with unnecessary toxicity and a delay in surgery.

Early systemic treatment may be effective to reduce distant relapse and prevent dissemination of micrometastases. Neoadjuvant chemotherapy (NAC) without radiation for LARC has recently been explored to reduce distant relapse and avoid the toxicities of radiation without compromising local control [6-8]. Although a few randomized, controlled studies are ongoing, there is no literature comparing NACRT and NAC performed at a single institution where the surgical technique for LARC is relatively homogeneous [9-11].

In the present study, different neoadjuvant treatment strategies for LARC, short-term survival results, and oncological results at our institution were compared.

\section{Methods}

\section{Patients and specimens}

This study was undertaken with the approval of the Institutional Review Board (IRB) for the protection of human subjects at the Dokkyo Medical University, Koshigaya Hospital.

A total of 55 patients (33 men, 22 women) with LARC treated with or without NAT followed by surgical resection at our institution between April 2010 and February 2016 were included. NAC and NACRT were performed in 27 and 28 patients, respectively. The median follow-up periods for relapse-free survival (RFS) and overall survival (OS) were 41.3 and 45.4 months, respectively. The eligibility criteria included (1) previously untreated, histologically confirmed, locally advanced adenocarcinoma of the rectum with or without nodal involvement and requiring surgery; (2) stage cT3/4 and cN + cM0; (3) World Health Organization (WHO) performance status $0-1$; and (4) age $<80$ years. Patients with clinical obstruction underwent temporary diverting ostomies.

All patients were initially evaluated by colonoscopy. To obtain an accurate diagnosis and investigate the Kirsten rat sarcoma viral oncogene homolog (KRAS) mutation state, multiple sites (4-7 sites) of the primary tumor were sampled and necrotic tissues were avoided. Pelvic magnetic resonance imaging (MRI) was used to determine the local extent of the disease, including the depth of invasion and lymph node metastases. When it was not possible to precisely determine nodal status on MRI, clinical nodal status was considered node-negative (N-negative) if there were no perirectal and lateral pelvic lymph nodes (LPLNs) with diameter $<7 \mathrm{~mm}$ and node-positive (N-positive) if there were one or more perirectal and lateral pelvic lymph nodes with diameter $\geq 7 \mathrm{~mm}$.

On MRI, tumor and lymph node shrinkage rates were defined respectively as $\mathrm{T}$ - and $\mathrm{N}$-downstaging, and shrinkage was defined as a decrease in size $\geq 30 \%$ in the comparison before and after NAT. Radiologic tumor response was evaluated in accordance with the Response Evaluation Criteria in Solid Tumors guidelines [12]. Chest- and abdominopelvic-computed tomography (CT) were used to diagnose distant metastases. Clinicopathological findings were analyzed based on the TNM classification of malignant tumors, according to the Union for International Cancer Control (UICC) [12]. Histological tumor regression after NAT was graded according to the Japanese Society for Cancer of the Colon and Rectum: grade 0 , no regression; grade 1a, minimal effect (necrosis less than one third of the lesion); grade $1 \mathrm{~b}$, mild effect (necrosis less than two thirds but one third or more of the lesion); grade 2 , moderate effect (necrosis more than two thirds of the lesion); and grade 3 , absence of residual tumor cells [13]. For convenience, grades $0,1 \mathrm{a}$, and $1 \mathrm{~b}$ were classified as non-responders and grades 2 and 3 were classified as responders.

\section{Neoadjuvant therapy}

The NACRT regimen consisted of external beam radiation (45 Gy delivered in 25 fractions) to the whole pelvis including the pelvic sidewall and 5-fluorouracil (5-FU)-based oral chemotherapy. Patients underwent surgical resection with curative intent 6-8 weeks after the completion of CRT. The NAC regimen was determined by the tumor histology including KRAS mutations. If patients had wild-type KRAS, 2 cycles of SOX: S-1 $\left(80 \mathrm{mg} / \mathrm{m}^{2}\right)$ on days 1 to 14 , oxaliplatin $\left(85 \mathrm{mg} / \mathrm{m}^{2}\right)$ on day 8 , plus cetuximab $\left(400 \mathrm{mg} / \mathrm{m}^{2}\right.$ as an initial weekly dose, followed by $250 \mathrm{mg} / \mathrm{m}^{2} /$ week) were given. If patients had a non-wild-type KRAS, 1-2 cycles of 2 cycles of SOX, 2-9 cycles of mFOLFOX6 in 2-week cycles (oxaliplatin, $85 \mathrm{mg} / \mathrm{m}^{2}$, L-leucovorin $200 \mathrm{mg} / \mathrm{m}^{2}$, 5-fluorouracil bolus 400 and $2400 \mathrm{mg} / \mathrm{m}^{2}$ continuous infusion over $46 \mathrm{~h}$ ), or 2-3 cycles of XELOX, with capecitabine $\left(1000 \mathrm{mg} / \mathrm{m}^{2}\right)$ twice daily on days $1-14$ and oxaliplatin $\left(130 \mathrm{mg} / \mathrm{m}^{2}\right)$ on day 1 , were given. Patients underwent surgical resection with curative intent 46 weeks after completion of NAC. Toxicity was assessed before each 2-3-week cycle, according to the National Cancer Institute Common Terminology Criteria for Adverse Events, version 4.0. 


\section{Surgery}

TME with/without sphincter preservation was to be carried out whenever feasible, according to the standardized techniques, using a laparoscopic or open approach. Laparoscopic and open approaches were performed in 39 (71\%) patients and 16 (29\%) patients, a sphincter-preserving operation (low anterior resection) was performed in 27 (49\%) patients, and an abdominoperineal resection (APR) was performed in 28 (51\%) patients, respectively. Creation of a temporary diverting ostomy was depended on the discretion of the primary surgeon. Lateral pelvic lymph node dissection (LPLND) was added if there was suspicious node positivity at the area of the pelvic sidewall based on a pelvic MRI before NAT.

The primary and secondary endpoints of the present study were 3-year RFS and local and distant relapse rates.

\section{Postoperative adjuvant therapy and follow-up evaluations}

Postoperative adjuvant chemotherapy was usually recommended to patients with ypT4 and ypN+ or those with positive circumferential resection margin for a total of 6 months including the duration of NAC and NACRT using a regimen including 5-FU-leucovorin, capecitabine, SOX, or FOLFOX. However, the selection of PAC of regimen was basically depended on the primary surgeon.
Patients had regular follow-up every 3-4 months, including clinical evaluations and physical examinations at least 5 years after surgery. Contrast-enhanced CT scans of the chest/abdomen and pelvis were performed every 6 months, and proctoscopy were performed every 1 year. Local and distant relapse was diagnosed by clinical evaluation and imaging.

\section{Statistical analysis}

Categorical variables are presented as numbers (percentage) and were analyzed with $\chi^{2}$ and Fisher's exact tests, and continuous variables are presented as medians (range) and analyzed with the Mann-Whitney $U$ test. RFS and OS curves were plotted using the Kaplan-Meier method and compared using the log-rank test. Statistical analysis was performed using SPSS version 23 (IBM Japan Ltd., Tokyo, Japan). A $p$ value less than 0.05 was considered significant.

\section{Results}

Baseline demographic and cancer characteristics

Patients who received NAC and NACRT showed similar demographic characteristics except for operation approach and operation method (Table 1). A significantly higher proportion of patients with NAC underwent laparoscopic surgery and anterior resection $(p=0.037$ and $p=0.003$,

Table 1 Baseline demographics and cancer characteristics

\begin{tabular}{|c|c|c|c|}
\hline \multirow[b]{2}{*}{ Characteristics } & \multicolumn{2}{|c|}{ Treatment group } & \multirow[b]{2}{*}{$p$ value } \\
\hline & NAC (27) & NACRT (28) & \\
\hline Age, median (range), years & $66.0(40-79)$ & $68.0(42-78)$ & $p=0.64^{*}$ \\
\hline Gender (male), $n$ (\%) & $17(63 \%)$ & $16(57 \%)$ & $p=0.79$ \\
\hline Pretreatment serum CEA level, average (range), $\mathrm{ml} / \mathrm{dl}$ & $4.7(0.9-646)$ & $6.2(1.5-1244)$ & $p=0.26^{*}$ \\
\hline Tumor size, average (range), $\mathrm{cm}$ & $5.0(2-10)$ & $4.9(2-10)$ & $p=0.91^{*}$ \\
\hline Distance from anal verge $(<5 \mathrm{~cm}), n(\%)$ & $17(63 \%)$ & $19(68 \%)$ & $p=0.78$ \\
\hline \multicolumn{4}{|l|}{ Tumor differentiation, $n$ (\%) } \\
\hline Well, moderate & $26(96 \%)$ & $27(96 \%)$ & \multirow[t]{2}{*}{$p=1.00$} \\
\hline Muc & $1(3.7 \%)$ & $1(3.6 \%)$ & \\
\hline \multicolumn{4}{|l|}{ cT category, n (\%) } \\
\hline pT3 & $24(89 \%)$ & $22(71 \%)$ & \multirow[t]{2}{*}{$p=0.46$} \\
\hline pT4a & $3(11 \%)$ & $5(18 \%)$ & \\
\hline \multicolumn{4}{|l|}{ cN category, n (\%) } \\
\hline pN1 & $18(67 \%)$ & $17(61 \%)$ & \multirow[t]{2}{*}{$p=0.78$} \\
\hline $\mathrm{pN} 2$ & $9(33 \%)$ & $11(39 \%)$ & \\
\hline Operation approach (lap), n (\%) & $23(85 \%)$ & $16(57 \%)$ & $p=0.037$ \\
\hline \multicolumn{4}{|l|}{ Operative method, $n$ (\%) } \\
\hline Low anterior resection & $19(70 \%)$ & $8(29 \%)$ & \multirow[t]{2}{*}{$p=0.003$} \\
\hline Abdominoperineal excision & $8(30 \%)$ & $20(71 \%)$ & \\
\hline LPLN dissection (yes), $n$ (\%) & $9(33 \%)$ & $5(18 \%)$ & $p=0.16$ \\
\hline Postoperative adjuvant chemotherapy (yes), $n(\%)$ & $9(33 \%)$ & $12(43 \%)$ & $p=0.58$ \\
\hline
\end{tabular}

*Mann-Whitney $U$ analysis 
respectively). There were no significant differences in other clinicopathological characteristics including age, sex, pre-treatment serum CEA level, tumor size, distance from the anal verge, tumor differentiation, clinical- $\mathrm{T}$ and clinical-N category, LPLND, and postoperative adjuvant chemotherapy.

\section{Comparison of neoadjuvant therapeutic response with clinicopathological findings and toxicities}

Surgical site infection (SSI) occurred more frequently in patients with NACRT than in those with NAC (71 vs. $29 \%, p<0.001)$. The percentages of patients with lymph node yield (LNY) less than 12 in the NAC group and the NACRT group were 26 and 68\%, respectively ( $p=0.002$, Table 2$)$. Except for the abovementioned factors, there were no significant differences in almost all factors, including normalization of CEA values post NAT, RECIST evaluation, pathological grade, distance from anal verge after NAT, circumferential resection margin (CRM), lymphovascular invasion, improved invasion ( $\mathrm{pT}$ ), and lymph node metastasis $(\mathrm{pN})$, pLPLN metastasis, and ypStage.

Regardless of the kind of NAT, no patient experienced any grade 3-4 therapy-related adverse events. The frequent toxic events were grade 1 diarrhea in patients with NACRT and neutropenia in patients with NAC (Table 3).

\section{Comparisons of neoadjuvant therapeutic response with patient outcomes}

Eleven of 55 (20\%) patients who received NAT developed metastatic disease, with the first confirmed site indicated in Table 4. In the NAC and NACRT groups, 2 patients had local relapse (NAC group $7.4 \%$ and CRT group 7.1\%). On the other hand, 2 patients who received NAC and 5 patients who received NACRT had distant relapse (NAC group 7.4\% and NACRT group 18\%). With re-analyses excluding 21 patients who received PAC, no patients who received NAC had a distant relapse (Table 5).

Although there were no significant differences in 3-year RFS and 4-year OS between NAC and NACRT (3-year RFS 85.2 vs. $70.4 \%, p=0.279$, Fig. 1; 4-year OS 96.3 vs. $89.1 \%, p=0.145$, respectively, Fig. 2 ), in analyses excluding patients who received PAC, there was no significant difference in OS (4-year OS NAC 100\% vs. NACRT 93\%, $p=0.14$, Fig. 4 ), but there was a significant difference in RFS (3-year RFS NAC $94.4 \%$ vs. NACRT 63.2\%, $p=0.043$, Fig. 3).

In total, 5 patients (CRT 4 patients, NAC 1 patient) died of metastatic rectal cancer during follow-up (Fig. 4). Six patients with metastatic disease were alive, one of whom received radiotherapy for pelvic lymph node relapse and was cancer-free.

Table 2 The comparison of neoadjuvant therapeutic response to clinicopathological findings

\begin{tabular}{|c|c|c|c|}
\hline \multirow[b]{2}{*}{ Characteristics } & \multicolumn{2}{|c|}{ Treatment group } & \multirow[b]{2}{*}{$p$ value } \\
\hline & NAC (27) & NACRT (28) & \\
\hline Median follow-up period of RFS & 47.0 & 32.5 & $p=0.23^{*}$ \\
\hline Median follow-up period of OS & 51.0 & 35.0 & $p=0.23^{*}$ \\
\hline Normalization of CEA value post NAT (yes), $n(\%)$ & $7(54 \%)$ & $8(50 \%)$ & $p=0.57^{*}$ \\
\hline Distance from anal verge after NAT $(<5 \mathrm{~cm}), n(\%)$ & $15(56 \%)$ & $14(50 \%)$ & $p=0.79$ \\
\hline Pathological response (responder), $n(\%)$ & $9(33 \%)$ & $13(46 \%)$ & $p=0.23$ \\
\hline${ }^{*}$ Complete response & $1(3.7 \%)$ & $5(18 \%)$ & \\
\hline Circumferential margin (negative), $n(\%)$ & $26(93 \%)$ & $26(93 \%)$ & $p=0.51$ \\
\hline Lymphovascular invasion (present), $n$ (\%) & $15(56 \%)$ & $13(46 \%)$ & $p=0.34$ \\
\hline ypT stage (ypT0-2), n (\%) & $11(41 \%)$ & $12(43 \%)$ & $p=0.55$ \\
\hline ypN (absent), $n$ (\%) & $21(78 \%)$ & $21(75 \%)$ & $p=0.53$ \\
\hline \multicolumn{4}{|l|}{ Lymph node yield, $n$ (\%) } \\
\hline Less than 12 & $7(26 \%)$ & $19(68 \%)$ & $p=0.002$ \\
\hline 12 or more & $20(74 \%)$ & $9(32 \%)$ & \\
\hline pLPLN (present), $n(\%)$ & $1 / 9(11 \%)$ & $0 / 5$ & $p=0.95$ \\
\hline \multicolumn{4}{|l|}{ ypStage, $n(\%)$} \\
\hline ypStage0 (CR) & $1(3.7 \%)$ & $4(14 \%)$ & $p=0.59$ \\
\hline ypStage1 & $7(26 \%)$ & $6(21 \%)$ & \\
\hline ypStage2 & $13(48 \%)$ & $12(43 \%)$ & \\
\hline ypStage3 & $6(22 \%)$ & $6(22 \%)$ & \\
\hline
\end{tabular}


Table 3 Number and percentage of patients with most common grade 1-2 toxicities by common terminology criteria for adverse events

\begin{tabular}{lll}
\hline NAC & Grade & \\
Category of toxicity & Grade 1, $n(\%)$ & Grade 2, n (\%) \\
Neutropenia & $4(15 \%)$ & 0 \\
Thrombocytopenia & $1(3.7 \%)$ & 0 \\
Hepatic dysfunction & $1(3.7 \%)$ & 0 \\
Neuropathy & $2(7.4 \%)$ & 0 \\
Dermatopathy & 0 & $1(3.7 \%)$ \\
Vomiting & $1(3.7 \%)$ & 0 \\
NACRT & Grade & \\
Category of toxicity & Grade 1,n (\%) & Grade 2,n (\%) \\
Kidney dysfunction & $1(3.7 \%)$ & 0 \\
Radiation dermatitis & $1(3.7 \%)$ & 0 \\
Diarrhea & $2(7.4 \%)$ & 0 \\
\hline
\end{tabular}

\section{Discussion}

The present study compared the therapeutic effects of NAC and NACRT with regard to clinicopathological findings, therapeutic toxicities, relapses, and survival curves at a single institution. Only one study so far has been published on the comparison of these treatment strategies for LARC patients [14]. In the present study, although the numbers of patients treated either by NAC or NACRT were small, several interesting results were found with regard to perioperative factors. Anal sphincter preservation was more often possible after NAC than after NACRT, even though the distance between the distal margins of the tumor and the anal verge before treatment did not differ between patients treated with NAC and those treated with NACRT. SSI was less frequent after NAC than after NACRT. In addition, the distant relapse rate of patients who received NAC was low compared with that of NACRT (7.4 vs. 18\%), and there was a significant difference in 3-year RFS excluding patients who received postoperative adjuvant chemotherapy (NAC vs. NACRT; 94.4 vs. $63.2 \%, p=0.043$ ).

Local relapse rates did not differ between NAC and NACRT, and they were almost equal to the local relapse rates reported in previous trials [1,2]. Although local relapse has been the most significant concern in patients after curative resection of LARC, distant relapse has also attracted much attention in recent years [2-4, 15].

Table 4 Summary of postoperative relapses

\begin{tabular}{lll}
\hline \multirow{2}{*}{ Location } & \multicolumn{2}{l}{ Treatment group } \\
\cline { 2 - 3 } & NAC & NACRT \\
\hline Overall relapse (present), $n(\%)$ & $4(15 \%)$ & $7(25 \%)$ \\
Local relapse (present), $n(\%)$ & $2(7.4 \%)$ & $2(7.1 \%)$ \\
Distant relapse (pulmonary and liver), $n(\%)$ & $2(7.4 \%)$ & $5(18 \%)$ \\
\hline
\end{tabular}

Table 5 Summary of postoperative relapses excluding patients who received PAC $(n=34)$

\begin{tabular}{lll}
\hline Location & \multicolumn{2}{l}{ Treatment group } \\
\cline { 2 - 3 } & NAC $(n=18)$ & $\operatorname{NACRT~}(n=16)$ \\
\hline Overall relapse (present), $n(\%)$ & $1(5 \%)$ & $5(31 \%)$ \\
Local relapse (present), $n(\%)$ & $1(5 \%)$ & $2(13 \%)$ \\
Distal relapse (pulmonary and liver), $n(\%)$ & 0 & $3(19 \%)$ \\
\hline
\end{tabular}

Distant relapse was slightly less frequent after NAC than after NACRT. No significant differences in OS and RFS were found between NAC and NACRT.

In analyses excluding patients who received PAC for the purpose of eliminating the effect of PAC, no patients who received NAC had a distant relapse, and consequently, RFS was significantly longer after NAC than after NACRT. These results suggest that NAC may be at least equally useful to NACRT for the prophylaxis of not only local relapse, but also distant relapse in patients with LARC.

After both NAC and NACRT, lymph node metastasis was not found (ypN0) in approximately $75 \%$ of patients and an improvement of depth of invasion to ypT0-1 was achieved in approximately $40 \%$ of patients after both NAC and NACRT. Pathological lymph node metastasis is the strongest prognostic factor in patients with colorectal cancer. Recent studies have shown a reduction of number of LNY after NACRT because of the reduction of the size and number of lymph nodes (LNs) by NACRT, due to fibrosis or disappearance [16-18]. Furthermore, LNY is an important prognostic factor because a smaller LNY may result in critical failure in identifying metastatic LNs, and several recent studies have also shown that LNY is also an important prognostic indicator in patients who received NACRT [19-21]. Data from a Dutch nationwide trial on rectal cancer

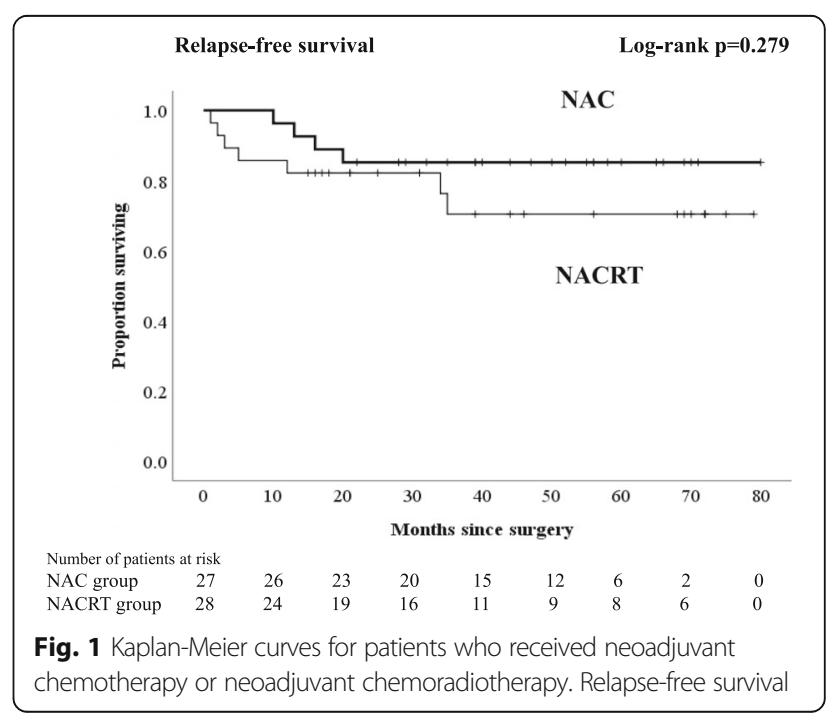




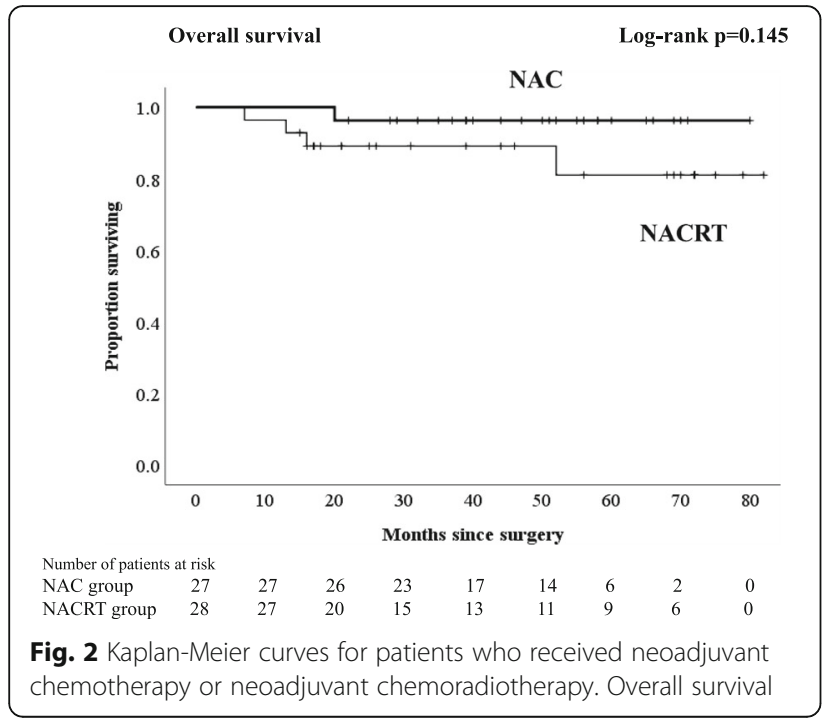

showed that 12 or more LNs were found in only $19 \%$ of patients who received NACRT [22]. In the present study, on routine pathological examination, the percentages of patients with 12 or more LNs in the NAC group and the NACRT group were 74 and $32 \%(p=0.002)$, respectively. These values were less than those in previous studies, especially in the NACRT group [20, 23-25]. Miller et al. reported that the mean LNY after NACRT was decreased by 7 to $53 \%$ compared with that without NACRT and that the mean value of the decrease in the studies used in their review was $28 \%$ [16]. In the present study, 3 of 14 (21\%) patients after NACRT with less than 12 LNs developed relapses. Therefore, the required number of LNs for the precise staging of patients after NACRT needs to be revised by further analyses.

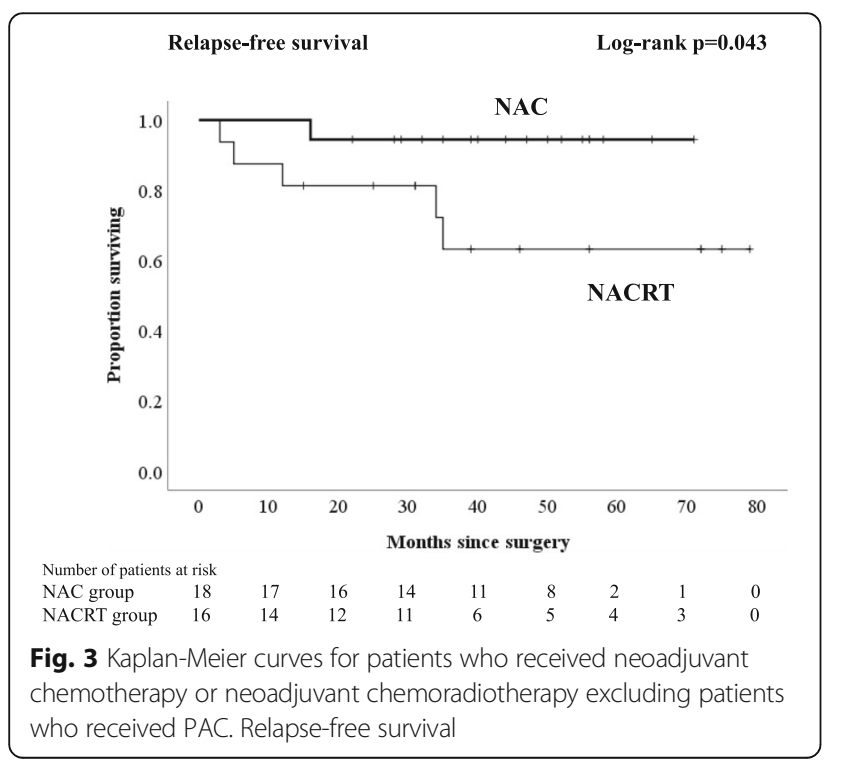

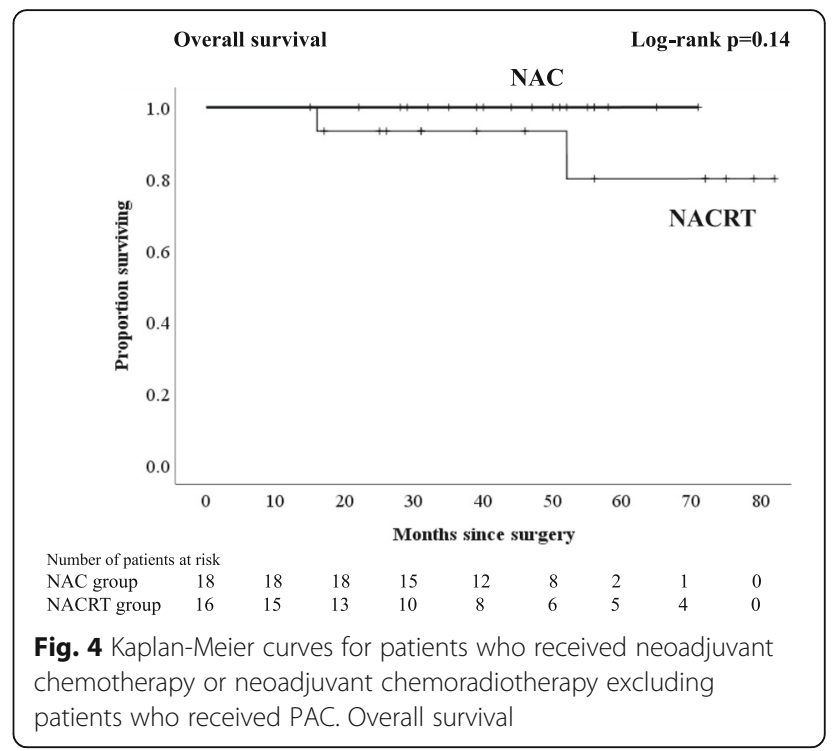

It has been generally acknowledged that NACRT increases the risk of treatment-related toxicities such as gastrointestinal, dermatologic, and neurologic toxicities in patients with LARC $[1,26]$. However, there were no grade 3/4 acute treatment-related toxicities in either the NACRT or the NAC group in the present study. Several trials of NACRT suggested that NACRT increased the incidence of surgical morbidity, especially perineal wound healing after APR $[27,28]$. In the present study, SSI was significantly more frequent in the NACRT group than in the NAC group (71 vs. $22 \%, p<0.001$ ). Therefore, neoadjuvant oxaliplatin-based chemotherapy appears to be a safe therapeutic option, and the risk of treatment-related toxicities and surgical complications does not exceed that of NACRT.

Currently, the PROSPECT study, comparing NAC alone and NAC plus NACRT (conventional CRT), is ongoing as a randomized phase II/III trial in North America with participation of all US and Canadian cooperative groups. More recently, the FOWARC study is also being carried out as a randomized phase III trial comparing NAC with or without radiation and NACRT (conventional CRT). Although disease-free survival (DFS) as the endpoint of the FOWARC study is still under evaluation, Deng et al. have concluded that NAC alone showed a lower $\mathrm{pCR}$ rate than NAC with radiation, but it had a similar downstaging rate to that of NACRT with less toxicity [9]. We should wait and evaluate their outcomes to improve the treatment cure for patents with LARC.

Studies comparing NAC and NACRT head-to-head for patients with LARC are very few. Cassidy et al. surveyed 21,707 patients who would meet the eligibility criteria of the PROSPECT trial from the National Cancer Data Base (NCDB) and compared NACRT and NAC (neoadjuvant 
multiagent chemotherapy) for clinical T2N1 (cT2N1), cT3N0, or cT3N1 rectal cancers [14]. Using propensity score-matched analysis to avoid selection bias, they observed a worse OS in the NAC group than in the NACRT group. They concluded that elimination of CRT for patients who have cT2N1, cT3N0, or cT3N1 rectal cancers should be avoided until the results of the PROSPECT trial are published. In Cassidy's study, however, the number of patients who received NAC alone was much less than that who received NACRT. The regimens and duration of NAC were not described and might have differed by institution and the period when the patients were treated. The patterns of relapse are not specified either. Therefore, the evaluation of NAC and NACRT for patients with LARC requires the common regimen including contents, duration, and postoperative treatment.

To the best of our knowledge, there has been no report from a single institution comparing NAC and NACRT, though the numbers of patients of both the NAC and NACRT groups were too small and the regimens of NAC were not homogeneous in the present study. The selection of either NAC or NACRT was not randomized and was usually based on the patient's preference. Surgeons' preference may also influence the selection of either of NAC and NACRT, because the patient's decision is often influenced by the surgeon's explanation when obtaining informed consent. In addition, some of the patients refused PAC by themselves despite the surgeons' recommendation based on the pathological findings. Therefore, no conclusion can be drawn from the present study alone. However, the results suggest that future prospective studies to explore the safety and efficacy of NAC for LARC would be valid.

\section{Conclusions}

In conclusion, the present study suggests that the therapeutic effect of oxaliplatin-based NAC appears equal to, or may be even greater than that of, NACRT in several clinicopathological factors such as reduced toxicity, increased number of LNY, and improved RFS in patients who did not received PAC. Although we are unable to specify the best regimen and duration of NAC from the present study, oxaliplatin-based NAC might be effective for both local and distant control of LARC. Further studies regarding the regimen and duration of NAC is warranted in order to identify the best method of NAC for LARC.

\footnotetext{
Abbreviations

APR: Abdominoperineal resection; CEA: Carcinoembryonic antigen; CRM: Circumferential resection margin; CT: Computed tomography; DFS: Disease-free survival; LARC: Locally advanced rectal cancer; LN: Lymph node; LNY: Lymph node yield; LPLND: Lateral pelvic lymph node dissection; MRI: Magnetic resonance imaging; NAC: Neoadjuvant chemotherapy; NACRT: Neoadjuvant chemoradiotherapy; NAT: Neoadjuvant therapy; NCDB: National Cancer Data Base; OS: Overall survival; PAC: Postoperative adjuvant chemotherapy; RECIST: Response Evaluation Criteria in Solid
}

Tumors; RFS: Relapse-free survival; SSI: Surgical site infection; TME: Total mesorectal excision; UICC: Union for International Cancer Control; WHO: World Health Organization

\section{Availability of data and materials}

The datasets used and/or analyzed during the current study are available from the corresponding author on reasonable request.

\section{Authors' contributions \\ TO participated in the design of this study, the surgical procedures, and the data analysis. SS, ET, RY, YY, NT, and MO participated in the surgical procedures. YO participated in the pathological diagnosis. All authors read and approved the final manuscript.}

\section{Ethics approval and consent to participate}

This study was approved by the local ethic committee, and written informed consent was obtained from every participant.

\section{Competing interests}

The authors declare that they have no competing interests.

\section{Publisher's Note}

Springer Nature remains neutral with regard to jurisdictional claims in published maps and institutional affiliations.

\section{Author details}

'Department of Surgery, Dokkyo Medical University, Koshigaya Hospital, 2-1-50, Minami Koshigaya, Koshigaya, Saitama 343-8555, Japan. ²Department of Pathology, Dokkyo Medical University, Koshigaya Hospital, 2-1-50, Minami Koshigaya, Koshigaya, Saitama 343-8555, Japan.

Received: 30 October 2017 Accepted: 22 May 2018

Published online: 05 June 2018

\section{References}

1. Sauer R, Becker H, Hohenberger W, Rodel C, Wittekind C, Fietkau R, et al. Preoperative versus postoperative chemoradiotherapy for rectal cancer. $\mathrm{N}$ Engl J Med. 2004;351:1731-40.

2. Sauer R, Liersch T, Merkel S, Fietkau R, Hohenberger W, Hess C, et al. Preoperative versus postoperative chemoradiotherapy for locally advanced rectal cancer: results of the German CAO/ARO/AIO-94 randomized phase III trial after a median follow-up of 11 years. J Clin Oncol. 2012;30:1926-33.

3. van Gijn W, Marijnen CA, Nagtegaal ID, Kranenbarq EM, Putter H, Wiggers T, et al. Preoperative radiotherapy combined with total mesorectal excision for resectable rectal cancer: 12-year follow-up of the multicenter, randomized controlled TME trial. Lancet Oncol. 2011;12:575-82.

4. Fokas E, Liersch T, Fietkau R, Hohenberger W, Beissbarth T, Hess C, et al. Tumor regression grading after preoperative chemoradiotherapy for locally advanced rectal carcinoma revisited: updated results of the CAO/ARO/AIO94 trial. J Clin Oncol. 2014;32:1554-62.

5. Fisher B, Wolmark N, Rockette H, Redmond C, Deutsch M, Wickerham DL, et al. Postoperative adjuvant chemotherapy or radiation therapy for rectal cancer: results from NSABP protocol R-01. J Natl Cancer Inst. 1988;80:21-9.

6. Uehara K, Hiramatsu K, Maeda A, Sakamoto E, Inoue M, Kobayashi S, et al. Neoadjuvant oxaliplatin and capecitabine and bevacizumab without radiotherapy for poor-risk rectal cancer: N-SOG 03 phase II trial. Jpn J Clin Oncol. 2013;43:964-71

7. Hasegawa J, Nishimura J, Mizushima T, Miyata Y, Kim HM, Takemoto H, et al. Neoadjuvant capecitabine and oxaliplatin (XELOX) combined with bevacizumab for high-risk localized rectal cancer. Cancer Chemother Pharmacol. 2014;73:1079-87.

8. Schrag D, Weiser MR, Goodman KA, Gonen M, Hollywood E, Cercek A, et al. Neoadjuvant chemotherapy without routine use of radiation therapy for patients with locally advanced rectal cancer: a pilot trial. J Clin Oncol. 2014; 32:513-8.

9. Deng Y, Chi P, Lan P, Wng L, Chen W, Cui L, et al. Modified FOLFOX6 with or without versus fluorouracil and leucovorin with radiation in neoadjuvant treatment of locally advanced rectal cancer: initial results of the Chinese FOWARC multicenter, open-label, randomized three-arm phase III trial. J Clin Oncol. 2016;34:3300-7. 
10. Chemotherapy alone or chemotherapy plus radiation therapy in treating patients with locally advanced rectal cancer undergoing surgery (PROSPECT). http//clinicaltrials.gov.

11. Bevacizumab and combination chemotherapy in rectal cancer until surgery (BACCHUS). http//clinicaltrials.gov.

12. Edge SB, Compton CC. American Joint Committee on Cancer (AJCC) cancer staging manual. 7th ed. New York: Springer; 2010.

13. Japanese Society for Cancer of the Colon and rectum. Japanese Classification of Colorectal Carcinomas. 2nd English ed. Tokyo: Kanehara; 2009.

14. Cassidy RJ, Liu Y, Patel K, Zhong J, Steuer CE, Kooby DA, et al. Can we eliminate neoadjuvant chemoradiotherapy in favor of neoadjuvant multiagent chemotherapy for select stage II/III rectal adenocarcinomas: analysis of the National Cancer Data base. Cancer. 2017:123:783-93.

15. Sun $Y$, Lin $H, L u X$, Huang $Y, X u Z$, Huang $S$, et al. A nomogram to predict distant metastasis after neoadjuvant chemoradiotherapy and radical surgery in patients with locally advanced rectal cancer. J Surg Oncol. 2017;115:462-9.

16. Miller ED, Robb BW, Cumming OW, Johnstone PA. The effect of preoperative chemoradiotherapy on lymph node sampling in rectal cancer. Dis Colon Rectum. 2012;55:1002-7.

17. Amajoyi R, Lee Y, Recio PJ, Kondylis PD. Neoadjuvant therapy for rectal cancer decreases the number pf lymph nodes harvested in operative specimens. Am J Surg. 2013;205:289-92.

18. Shvero J, Koren R, Marshak G, Sadov R, Hadar T, Yaniv E, et al. Histological changes in the cervical lymph nodes after radiotherapy. Oncol Rep. 2001;8:909-11.

19. Mekenkamp LJ, van Krieken JH, Marijnen CA, van de Veldo CJ, Nagtegaal ID, et al. Lymph node retrieval in rectal cancer is dependent on many factors - the role of the tumor, the patient, the surgeon, the radiotherapist, and the pathologist. Am J Surg Pathol. 2009;33:1547-53.

20. Ha YH, Jeong SY, Lim SB, Choi HS, Chang HJ, Kim DY, et al. Influence of preoperative chemoradiotherapy on the number of lymph nodes retrieved in rectal cancer. Ann Surg. 2010;252:336-40.

21. Xu Z, Bertho ME, Becerra AZ, Aquina CT, Hensley BJ, Arsalanizadeh R, et al. Lymph node yield is an independent predictor of survival in rectal cancer regardless of receipt of neoadjuvant therapy. J Clin Pathol. 2017;70:584-92.

22. Nagtegaal ID, van Krieken JHJM. Role of pathologists in quality control of diagnosis and treatment of rectal cancer. Eur J Cancer. 2002;38:964-72.

23. De Campos-Lobato LF, Stocchi L, de Sousa JB, Buta M, Lavery IC, Fazio WW, et al. Less than 12 nodes in the surgical specimen after total mesorectal excision following neoadjuvant chemoradiation: it means more than you think! Ann Surg Oncol. 2013;20:3398-406.

24. Lykke J, Jess P, Danish Colorectal Cancer Group. Increased lymph node yield is associated with improved survival in rectal cancer irrespective of neoadjuvant treatment: results from a national cohort study. Dis Colon Rectum. 2015;58:823-30.

25. Gurawalia J, Dev K, Nayak SP, Kurpad V, Pandey A. Less than 12 lymph nodes in the surgical specimen after neoadjuvant chemo-radiotherapy: an indicator of tumor regression in locally advanced rectal cancer? J Gastrointest Oncol. 2016;7:946-57.

26. Loos M, Quentmeier P, Schuster T, Nitsche U, Gerther R, Keerl A, et al. Effect of preoperative radio(chemo)therapy on long-term functional outcome in rectal cancer patients: a systematic review and meta-analysis. Ann Surg Oncol. 2013;20:1816-28.

27. Marijinen CA, Kapiteijn E, van de Velde CJ, Martijn H, Steup WH, Wiggers $T$, et al. Acute side effects and complications after short-term preoperative radiotherapy combined with total mesorectal excision in primary rectal cancer: report of a multicenter randomized trial. J Clin Oncol. 2002;20:817-25.

28. Birgisson H, Pahlman L, Gunnarsson U, Glimelius B. Adverse effects of preoperative radiation therapy for rectal cancer: long-term follow-up of the Swedish Rectal Cancer Trial. J Clin Oncol. 2005:23:8697-705.

Ready to submit your research? Choose BMC and benefit from:

- fast, convenient online submission

- thorough peer review by experienced researchers in your field

- rapid publication on acceptance

- support for research data, including large and complex data types

- gold Open Access which fosters wider collaboration and increased citations

- maximum visibility for your research: over $100 \mathrm{M}$ website views per year

At BMC, research is always in progress.

Learn more biomedcentral.com/submissions 